\title{
Mucosal large cell neuroendocrine carcinoma of the head and neck regions in Japanese patients: a distinct clinicopathological entity
}

\author{
Kimihide Kusafuka, ${ }^{1}$ Masato Abe, ${ }^{1}$ Yoshiyuki lida, ${ }^{2}$ Tetsuro Onitsuka, ${ }^{2}$ Tomohito Fuke, ${ }^{2}$ \\ Rei Asano, ${ }^{2}$ Tomoyuki Kamijo, ${ }^{2}$ Takashi Nakajima ${ }^{1}$
}

${ }^{1}$ Pathology Division, Shizuoka Cancer Center Hospital and Research Institute, Shizuoka, Japan

${ }^{2}$ Division of Head and Neck Surgery, Shizuoka Cancer Center Hospital and Research Institute, Shizuoka, Japan

\section{Correspondence to}

Dr Kimihide Kusafuka, Pathology Division, Shizuoka Cancer Center Hospital and Research Institute, 1007 Shimonagakubo, Nagaizumi-cho, Sunto-gun, Shizuoka 411-8777, Japan; k.kusafuka@scchr.jp

Accepted 10 April 2012

\section{UNLOCKA}

This paper is freely available online under the BMJ Journals unlocked scheme, see http:// jcp.bmi.com/site/about/ unlocked.xhtml

\begin{abstract}
Backgrounds Large cell neuroendocrine carcinoma (LCNEC) is well-known as a lung cancer subtype. This study assessed the prevalence of head and neck mucosal LCNEC (M-LCNEC).
\end{abstract}

Methods M-LCNEC was studied clinically, histologically and immunohistochemically.

Results 0 f 814 surgically resected cases of mucosal head and neck carcinoma, only eight cases $(0.98 \%$; all men, mean age 64.6 years) were rediagnosed as M-LCNEC. They occurred in the oropharynx $(n=3)$, larynx $(n=4)$ and hypopharynx $(n=1)$. Seven of the cases had regional lymph node metastases and four resulted in death. Histologically, M-LCNEC had a sheet-like trabacular organoid growth pattern of relatively large basaloid cells in which central necrosis, rosette formation, peripheral palisading and high mitotic figures were evident. M-LCNEC was immunopositive for two or three neuroendocrine markers (CD56, chromogranin-A and synaptophysin). All cases showed high proliferative activity.

Conclusion M-LCNEC in the head and neck regions is a distinct histopathological entity whose positivity for neuroendocrine markers makes its diagnosis important. As about half of the patients died of the disease, M-LCNEC has a relatively poor prognosis.

\section{INTRODUCTION}

Large cell carcinoma is a well-known lung cancer subtype. Large cell neuroendocrine carcinoma (LCNEC) is an organoid growth pattern of large atypical cells which are immunopositive for more than one kind of neuroendocrine (NE) marker including CD56, chromogranin-A (ChA) and synaptophysin (Syn). ${ }^{1}$ Clinically, LCNEC of the lungs usually preludes a poor prognosis. ${ }^{2}$ LCNEC has also been reported in other organs such as the ovary, ${ }^{3-5}$ stomach $^{67}$ and urinary bladder, ${ }^{89}$ which similarly heralds a poor prognosis.

To the best of our knowledge, only five cases of LCNEC in the head and neck regions arising from the parotid gland have been reported in the English literature, ${ }^{10-13}$ as well as one case of LCNEC arising from the tongue base mucosa. ${ }^{14} \mathrm{~A}$ recent report described 10 cases (six new cases and four previously reported cases) of laryngeal LCNEC. ${ }^{15}$ The present study was undertaken to assess the lesion concept of LCNEC arising from the mucosa of the head and neck regions in Japanese patients and to describe the clinicopathological features of mucosal LCNEC (M-LCNEC).

\section{MATERIALS AND METHODS}

Eight hundred and fourteen surgically resected cases of mucosal cancer of the head and neck regions were identified from 2002 to 2009 in the pathology files of Shizuoka Cancer Center Hospital, Shizuoka, Japan. All were primary surgically resected Japanese patients. Twenty-four cases of basaloid squamous cell carcinoma (BSCC) and 73 cases of poorly differentiated squamous cell carcinoma (PDSCC) were selected; 24 BSCC and 32 PDSCC were extracted according to the histological criteria of pulmonary LCNEC in the WHO classification. ${ }^{1}$ Clinical data of all cases were obtained. Immunohistochemical examinations for CD56 (neural cell adhesion molecule, NCAM), ChA and Syn as NE markers were performed with an EnVision kit (DakoCytomation, Carpinteria, California, USA). The antibodies used in this study are summarised in table 1. Peroxidase activity was detected using 3-3'-daminobenzidine as the chromogen. After the sections were counterstained with haematoxylin, they were dehydrated and mounted in the synthetic medium. Immunopositivity of all tumour cells examined in a single section of $<10 \%, 10-19 \%, 20-49 \%$ and $>50 \%$ was defined as focally + , partially,++ and ++ , respectively. Staining evaluation was performed using semiquantitative optical analysis by two independent observers (KK and MA). Cases that were immunopositive for two or three NE markers were defined as M-LCNEC and were subsequently examined in detail for their clinical, histological and immunohistochemical characteristics.

Transmission electron microscopy was performed in one patient (case 3) using formalin-fixed specimens with decalcification. Small blocks (approximately $1 \mathrm{~mm}^{3}$ ) of resected specimens were postfixed in $2 \%$ glutaraldehyde in phosphate buffered saline at $4^{\circ} \mathrm{C}$ overnight and then for $2 \mathrm{~h}$ at $4^{\circ} \mathrm{C}$ with $1 \%$ osmium tetraoxide in phosphate buffered saline. Following dehydration, each specimen was transferred to propylene oxide and embedded in Quetol 812 epoxy resin (Nisshin EM, Tokyo, Japan). Ultrathin sections were cut with an Ultracut microtome (Leica, Weltzlar, Germany) and stained with uranyl acetate and lead citrate. Sections were observed with a JEM-1230 electron microscope (JEOL, Tokyo, Japan) using standard operating conditions.

\section{RESULTS}

Among the 814 surgically resected patients with mucosal cancer of the head and neck regions, eight cases $(0.98 \%)$ of M-LCNEC were retrospectively 
Table 1 Antibodies used in the study

\begin{tabular}{|c|c|c|c|c|}
\hline Antigen & Clone & $\begin{array}{l}\text { Antigen } \\
\text { retrieval }\end{array}$ & $\mathbf{P} / \mathbf{M}$ & Source \\
\hline CD56 (NCAM) & $\begin{array}{l}\text { NCC- } \\
\text { Lu-243 }\end{array}$ & Autoclave* & M & Nippon Kayaku (Tokyo, Japan) \\
\hline Chromogranin-A & & & $\mathrm{P}$ & $\begin{array}{l}\text { DakoCytomation (Carpinteria, } \\
\text { California, USA) }\end{array}$ \\
\hline Synaptophysin & & & $\mathrm{P}$ & $\begin{array}{l}\text { Signet Laboratories (Dedham, } \\
\text { Massachusetts, USA) }\end{array}$ \\
\hline TTF-1 & 8G7G3/1 & Autoclave* & M & DakoCytomation \\
\hline p63 & $4 \mathrm{~A} 4$ & Autoclave $^{*}$ & $\mathrm{M}$ & $\begin{array}{l}\text { LAB Vision (Fremont, } \\
\text { California, USA) }\end{array}$ \\
\hline HMWK & 34betaE12 & Autoclave* & M & DakoCytomation \\
\hline $\mathrm{N}$-cadherin & & Autoclave $\dagger$ & $\mathrm{P}$ & TaKaRa Bio (Shiga, Japan) \\
\hline Ki-67 & MIB-1 & Autoclave* & $\mathrm{M}$ & DakoCytomation \\
\hline
\end{tabular}

identified from the pathology files. The clinicopathological data are summarised in table 2.

\section{Clinical findings}

All eight cases of M-LCNEC were men (mean age 64.6 years, range 52-75) and all were smokers. The main complaints were dysphagia, dyspnoea and an abnormal feeling in the throat. M-LCNEC occurred at the base of the tongue $(n=2)$, lateral wall of the oropharynx $(n=1)$, larynx $(n=4 ; 3$ supraglottis and one infraglottis) and hypopharynx $(n=1$; posterior wall). Pathological TNM classification was T2 $(n=5), T 3(n=2)$, T4a $(n=1)$, N0 $(n=1), N 1(n=1), N 2 b(n=3)$ and N2c $(n=3)$. Seven of the eight cases of M-LCNEC had regional lymph node metastases. At surgery all cases showed no evidence of distant metastasis (M0). Two patients were treated with postoperative radiotherapy and four were treated with postoperative chemotherapy. One patient had been previously reported. ${ }^{14}$ This patient (case 2) had M-LCNEC at the tongue base and had previously had hepatocellular carcinoma and hypopharyngeal carcinoma which had been surgically resected. The hepatocellular carcinoma recurred but disappeared with radiotherapy. The oropharyngeal M-LCNEC also recurred and the patient died from uncontrolled locoregional recurrence of M-LCNEC. Hypopharyngeal carcinoma was moderately differentiated squamous cell carcinoma but was confirmed radiographically, surgically and pathologically to have no direct association with the cancer at the tongue base. Another patient (case 6) subsequently developed lung cancer and was treated with radiotherapy. The lung cancer, which was diagnosed as adenocarcinoma, was not associated with the laryngeal M-LCNEC and the patient survived. Two patients died from haemorrhage due to the progression of myelodysplastic syndrome (case 1) and multiple lung and liver metastases (case 3), respectively. Two other patients also died of uncontrolled locoregional recurrence. The remaining patients have been disease-free for follow-up periods of 24-108 months.

\section{Pathological findings}

Grossly, some tumours showed deep ulcerative lesions and endophytic growth with a greyish-white appearance of the cut surface, whereas other tumours-especially those of the larynx and hypopharynx-had a submucosal tumour-like appearance (figure 1).

Histologically, M-LCNEC had a solid or sheet-like trabecular and organoid growth pattern (figure 2A). The tumours had moderately to markedly desmoplastic stroma and were directly connected with the surface epithelium but showed no evidence of a carcinoma in situ component around the tumour. Rosette formation was frequently seen in the tumour nests (figure $2 \mathrm{~B}$ ). Some tumours had a basaloid pattern with peripheral palisading whereas others had central necrosis in the tumour nest (figure 2C,D). Squamoid differentiation was seen infrequently. The tumour cells had irregular polygonal cells with marked cellular atypia and relatively large cytoplasm which was moderately pleomorphic (figure 2A; inset). In some cases the tumour cells resembled basaloid cells. The nuclei of the tumour cells showed hyperchromatism and short spindle or smoothly ovoid shapes with a fine to coarsely granular chromatin pattern and frequently contained one large nucleolus. The mitotic rate was high (15-48 per 10 high power fields). Some tumours invaded the surrounding submucosal muscle layers and/or cartilages.

Immunohistochemically, M-LCNECs were positive for two or three of the NE markers CD56, ChA and Syn (figure 3A-C). All cases were immunopositive for CD56. Five cases (62.5\%) were immunopositive for $\mathrm{ChA}$ and six cases $(75.0 \%)$ were positive for Syn. Only two cases were immunopositive for thyroid transcription factor (TTF)-1 (figure 3D); these cases were immunopositive for all NE markers. Six cases (75.0\%) were immunopositive for $\mathrm{N}$-cadherin. Two cases showed diffuse immunopositivity for high molecular weight keratin. With the exception of cases 1 and 2, all cases had markedly decreased p63 immunopositivity areas (figure $3 \mathrm{E}$ ). All cases were p53 immunopositive. The Ki-67 labelling index (figure 3F) was high (mean $90.0 \%$, range $78.1-96.2 \%$ ).

In case 2 some normal basal cells adjacent to the tumour were immunopositive for CD56 and N-cadherin but negative for ChA and Syn (figure 4A,B).

\section{Ultrastructure}

Some tumour cells contained NE granules with dense cores of $250 \mathrm{~nm}$ in diameter in the cytoplasm (figure 5).

Table 2 Clinicopathological summary of cases of mucosal large cell neuroendocrine carcinoma

\begin{tabular}{|c|c|c|c|c|c|c|c|c|c|c|c|c|c|}
\hline Case no & Age/sex & Site & pTN & Tx & Prognosis (months) & CD56 & ChA & Syn & TTF-1 & p63 & HMWK & N-cad & Ki-67 LI \\
\hline 1 & $61 / \mathrm{M}$ & TB & $\mathrm{T} 2 \mathrm{~N} 2 \mathrm{c}$ & $S+R$ & DOAD (35 m) & $f+$ & - & ++ & - & ++ & ++ & - & $86.7 \%$ \\
\hline 2 & $65 / \mathrm{M}$ & TB & T2NO & S & DOD (50 m) & ++ & $f+$ & - & - & ++ & ++ & + & $90.8 \%$ \\
\hline 3 & $63 / \mathrm{M}$ & $\mathrm{LW}$ & $\mathrm{T} 2 \mathrm{~N} 2 \mathrm{~b}$ & $S+R$ & DOD (15 m) & ++ & ++ & ++ & ++ & - & - & ++ & $93.1 \%$ \\
\hline 4 & $69 / M$ & Larynx & T3N2b & $S+C$ & $\mathrm{DOD}(25 \mathrm{~m})$ & ++ & $p+$ & ++ & - & $f+$ & - & + & $78.1 \%$ \\
\hline 5 & $67 / M$ & Larynx & T4aN2c & $S+C$ & AWOD (31 m) & ++ & - & ++ & - & $\mathrm{p}+$ & $f+$ & ++ & $87.2 \%$ \\
\hline 6 & $74 / \mathrm{M}$ & Larynx & T2N2c & $\mathrm{S}$ & AWOD (18 m) & ++ & $f+$ & - & - & + & $f+$ & + & $95.3 \%$ \\
\hline 7 & $65 / \mathrm{M}$ & Larynx & $\mathrm{T} 3 \mathrm{~N} 2 \mathrm{~b}$ & $S+C$ & AWOD (24 m) & ++ & $f+$ & + & + & $f+$ & - & - & $96.2 \%$ \\
\hline 8 & $52 / \mathrm{M}$ & Нуро & $\mathrm{T} 2 \mathrm{~N} 1$ & $\mathrm{~S}+\mathrm{C}$ & AWOD $(90 \mathrm{~m})$ & ++ & - & ++ & - & $f+$ & - & + & $92.8 \%$ \\
\hline
\end{tabular}

AWOD, alive without disease; C, chemotherapy; ChA, chromogranin-A; DOAD, died of another disease; DOD, died of disease; HMWK, high molecular weight keratin; hypo, hypopharynx; Ki-67 LI, Ki-67 labelling index; LW, lateral wall of the oropharynx; N-cad, N-cadherin; R, radiotherapy; S, surgery; Syn, synaptophysin; TB, tongue base; TTF-1, thyroid transcription factor-1; Tx, treatment.

- , negative; $f+$, focally positive $(<10 \%$ positive); $p+$, partially positive $(10-19 \%$ positive); + , positive $(20 \%-49 \%$ positive $) ;++$, diffusely and strongly positive $(>50 \%$ positive). 


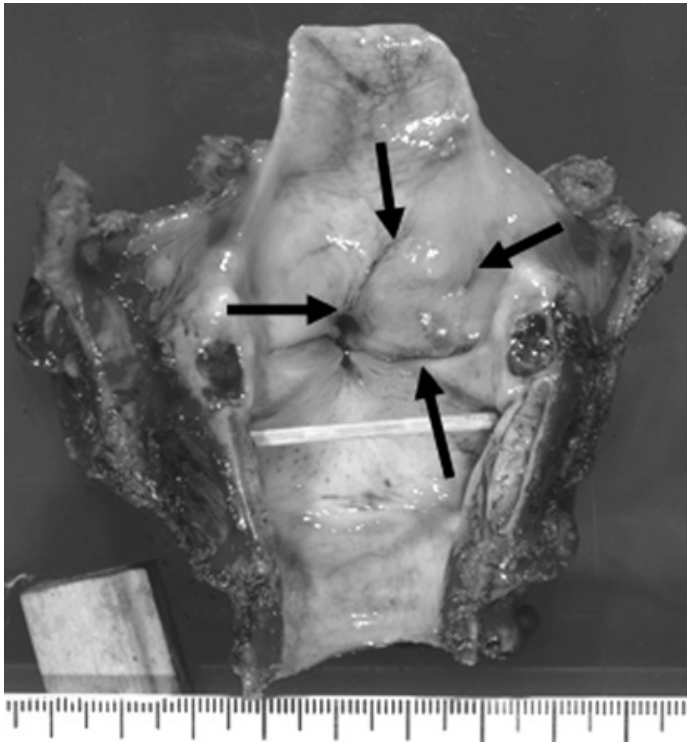

Figure 1 Laryngeal tumour (case 4) showed submucosal tumour-like appearance (arrows).

\section{DISCUSSION}

LCNEC was the first subtype of lung cancer extracted from large cell carcinoma, poorly differentiated adenocarcinoma and PDSCC of the lung. ${ }^{16}$ Subsequently, LCNECs have been reported in other organs including the ovary, ${ }^{3-5}$ stomach, ${ }^{6} 7$ urinary bladder $^{8} 9$ and parotid gland. ${ }^{10-13}$ LCNECs of both lungs and other organs lead to a poor prognosis because of the high-grade malignancy of the cancer.
In the head and neck region only five cases of LCNEC arising from the parotid gland have been reported, ${ }^{10-13}$ whereas 12 cases have been reported from the larynx (supraglottis) ${ }^{15} 17$ and from the base of the tongue. ${ }^{14}$ Thus, LCNECs are extremely rare in the head and neck region. In the larynx the majority of $\mathrm{NE}$ tumours are atypical carcinoid which clinically has an aggressive course. ${ }^{18-20}$ The detailed clinicopathological features of M-LCNEC of the head and neck region are unclear. Recently, Lewis et al reported that $88 \%$ of patients with laryngeal LCNEC died of the disease. M-LCNEC of the head and neck region therefore has a worse prognosis than carcinoid of the head and neck regions, ${ }^{15}$ and our Japanese series also indicates a relatively poor prognosis. We have recently reviewed LCNEC of the head and neck including M-LCNEC. ${ }^{21}$

In this histological review of 814 resected head and neck mucosal cancers, eight cases of M-LCNEC were identified with $\mathrm{NE}$ differentiation being confirmed immunohistochemically in the majority of the tumour cells. Morphologically, the tumours differed from both carcinoids and small cell carcinomas as reflected, to a certain extent, by their original diagnosis as BSCC or PDSCC. These results therefore clearly show that the spectrum of NE tumours of the head and neck region is broader than the previously recognised carcinoids and small cell carcinomas in the current World Health Organization ${ }^{19}$ and other widely used classifications. $^{22} 23$ The results indicate that M-LCNEC probably represents a distinct and independent pathological entity recognisable even in routine $\mathrm{H} \& \mathrm{E}$-stained specimens, although immunohistochemistry is necessary for confirmation. In the present study, M-LCNEC accounted for at least $0.98 \%$ of all head and neck mucosal cancers. Ferlito et al proposed that poorly differentiated NE carcinoma (grade III) of the larynx should be
Figure 2 Histological findings of mucosal large cell neuroendocrine carcinoma. (A) A representative tumour displaying recognisable organoid architectures and trabecular growth pattern, which prompted the correct diagnosis. In this area, relatively scanty stroma was observed. Inset: Tumour cells demonstrate relatively large eosinophilic cytoplasm and ovoid nuclei with coarse chromatin pattern and welldefined nuclear membranes (H\&E stain). (B) The tumour frequently displayed rosette formations (arrows) suggestive of neuroendocrine differentiation (H\&E stain). (C) Some tumours showed nests of basaloid pattern with peripheral nuclear palisading, similar to basaloid carcinoma of the lungs, but suggestive of neuroendocrine differentiation (H\&E stain). (D) The tumour frequently showed central necrosis in the solid nests and moderate to marked cellular atypia (H\&E stain).
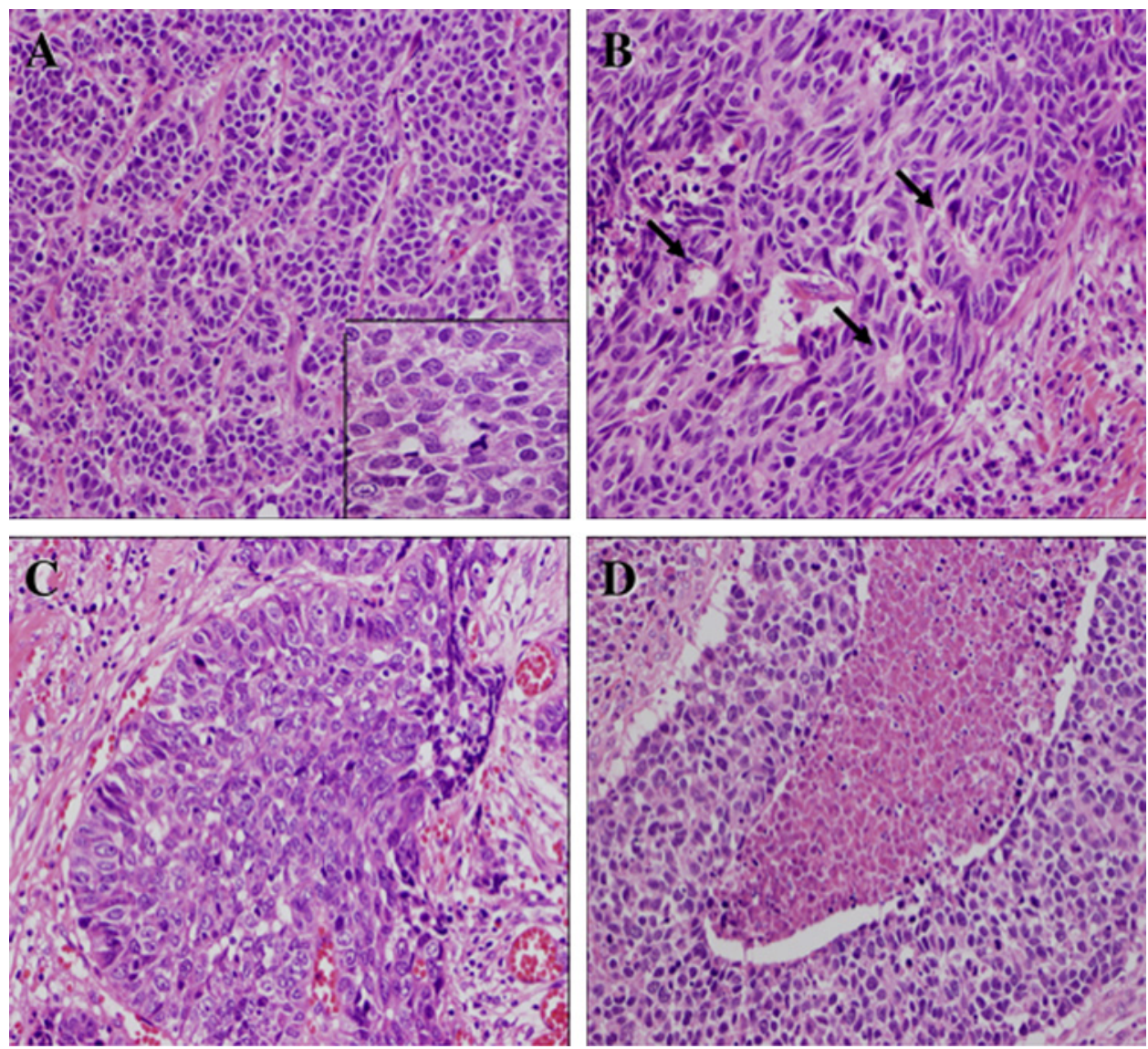
Figure 3 Immunohistochemical findings of mucosal large cell neuroendocrine carcinoma. The majority of the tumour cells are positive for $(A)$ CD56 (NCAM), (B) chromogranin-A and (C) synaptophysin. In case 3 the nuclei of the tumour cells were positive for TTF-1 (D) whereas in case 7 only small numbers of tumour cells were positive for p63 (arrows) (E). The nuclear labelling of Ki-67 (F) is markedly high, suggestive of high proliferative activity of the tumour cells but not that of welldifferentiated neuroendocrine tumours/ carcinomas.
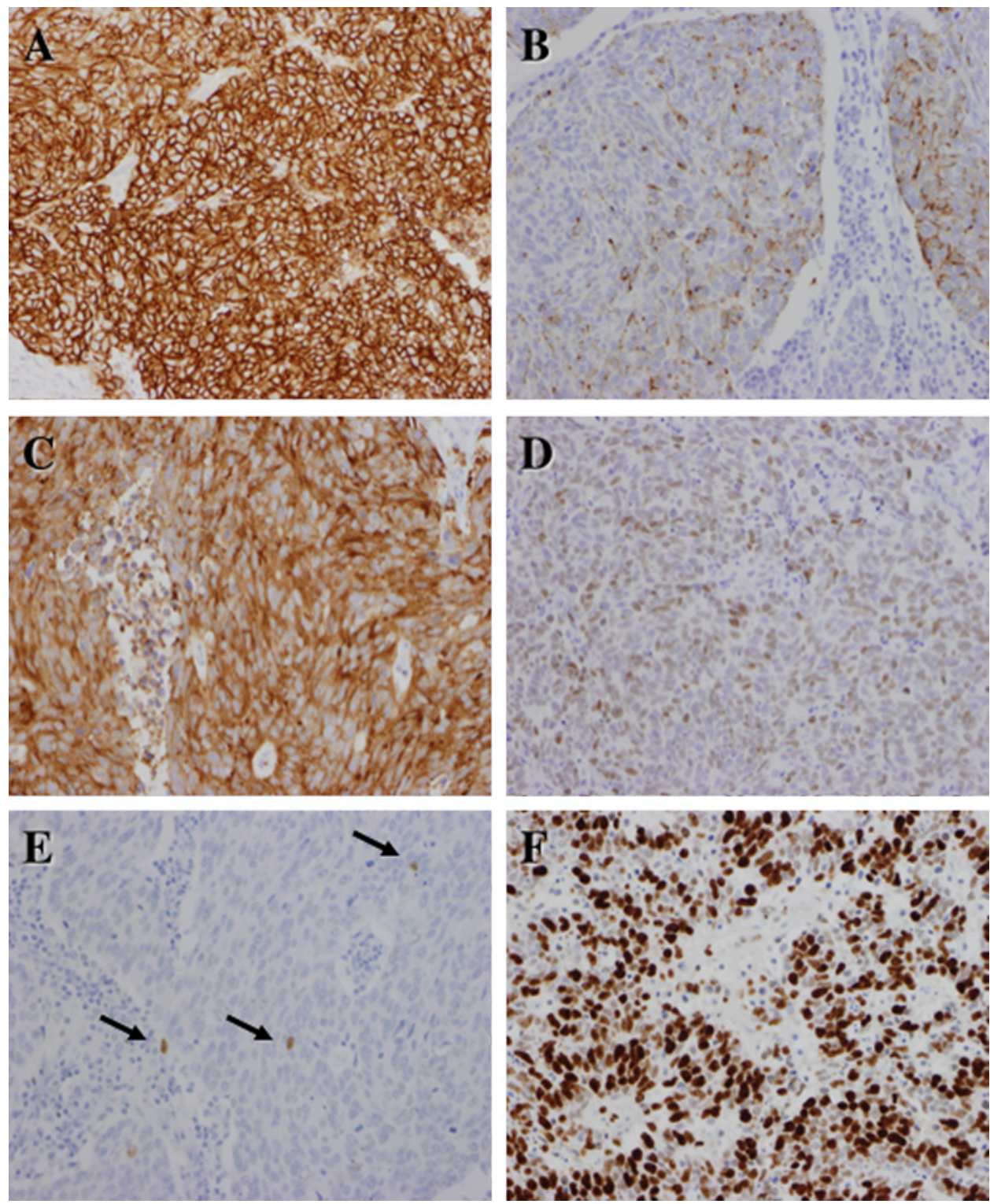

subclassified into small cell NE carcinoma and LCNEC, although the two clinical tumours usually have a poor prognosis. ${ }^{24}$

As with its pulmonary counterpart, the greatest difficulty with the diagnosis of M-LCNEC is how to recognise its NE morphology. Most of the present cases of M-LCNEC had solid growth and/or a basaloid pattern, and the most important issue was to distinguish them from BSCC. Organoid architecture was a good initial indication of M-LCNECs, even if it was faint and barely recognisable in most tumours, and the subsequent identification of a typical rosette formation by close scanning at higher magnification contributed to a correct diagnosis in most cases. In addition, careful evaluation of the cytological features included in the established histological criteria was also helpful in the differential diagnosis. In this study, most of the M-LCNECs were initially diagnosed as BSCC due to central necrosis and peripheral nuclear palisading, but careful histological observation frequently revealed an organoid architecture and rosette formation. Moreover, since the tumour cells cytologically resembled atypical basaloid cells, differential diagnosis between M-LCNEC and BSCC could be difficult. Unlike BSCC, M-LCNEC tumour cells frequently contain a relatively large and/or polygonal cytoplasm. Although it is sometimes difficult to distinguish M-LCNEC from BSCC from nuclear findings only, the typical nuclear findings of M-LCNEC show a fine to coarsely granular chromatin pattern with one large nucleolus. Histologically, it is most important to determine NE morphology such as organoid architecture and rosette formation. As M-LCNEC has little evidence of squamoid features such as single cell keratinisation and intercellular bridges, it is easy to distinguish from PDSCC. It is important to differentiate M-LCNEC from atypical carcinoid, especially in laryngeal tumours. Unlike atypical carcinoid, M-LCNEC has large cell size, cell pleomorphism, necrosis and high mitotic activity ( $>10 / 10$ high power fields). The major feature that distinguishes between M-LCNEC and atypical carcinoid is mitotic activity. ${ }^{15} 21$

Immunohistochemical examination of $\mathrm{NE}$ markers is very useful in the diagnosis of M-LCNEC, similar to pulmonary LCNEC. ${ }^{1}$ If lung cancers are immunopositive for at least one of the NE markers (CD56, ChA and Syn), the tumour can be diagnosed as LCNEC. In other head and neck tumours CD56 immunopositivity can occur. One study documented positive rates of $74 \%$ of ameloblastomas and $50 \%$ of keratocystic 

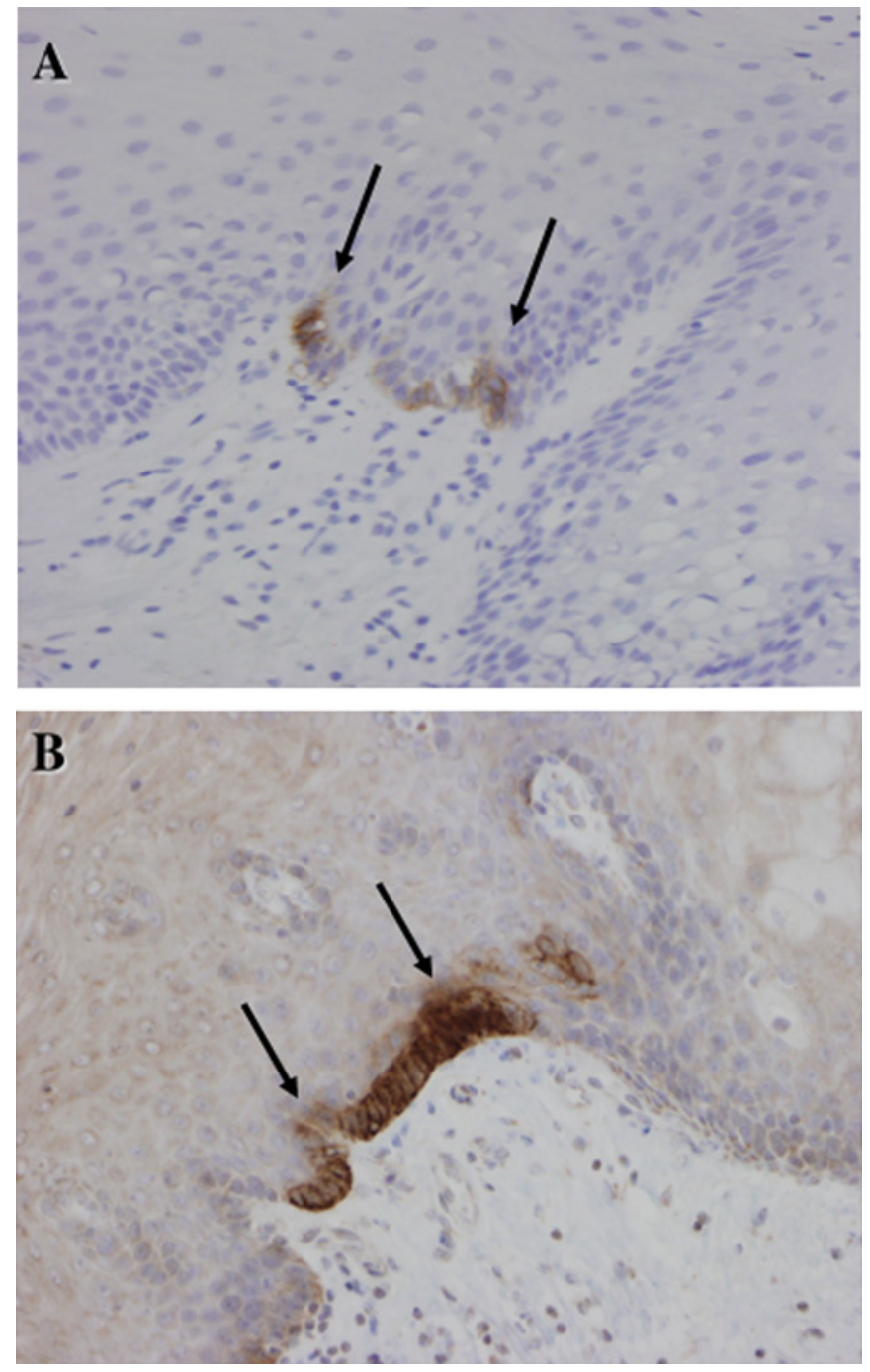

Figure 4 Immunostaining of case 3. Some normal basal cells in the squamous epithelium near the tumour are focally but strongly positive for (A) CD56 and (B) N-cadherin. Histologically, such cells cannot be distinguished from other basal cells.

odontogenic tumours of the jaw bones. ${ }^{25}$ Salivary LCNEC is immunopositive for ChA and/or Syn. ${ }^{10-12}$ Immunohistochemistry for ChA and Syn thus appears to be useful in the diagnosis of LCNEC. Appropriately, the present study defined M-LCNEC based on immunopositivity for two or three NE markers. In this study only two cases of M-LCNEC were immunopositive for TTF-1. In pulmonary LCNECs about half of the cases are immunopositive for TTF- $1{ }^{26}$ In M-LCNEC, aberrant TTF-1 expression is related to high-grade NE proliferations like pulmonary NE tumours, although TTF-1 expression is observed in other tumours such as pulmonary adenocarcinomas or thyroidal carcinomas.

Unlike both BSCCs and PDSCCs, almost all cases of M-LCNEC (except two) showed only focal immunopositivity for p63, which is a marker for basal cell and squamous cell differentiation. This finding echoes the published findings for basaloid carcinoma (one of the subtypes of pulmonary LCNEC) of the lungs in which $10-20 \%$ are immunopositive for both NE markers, p63 and high molecular weight keratin. ${ }^{27}$ The marked decrease in p63 expression and the increase in expression of $\mathrm{NE}$ molecules are therefore characteristic of M-LCNEC in the head and neck regions.

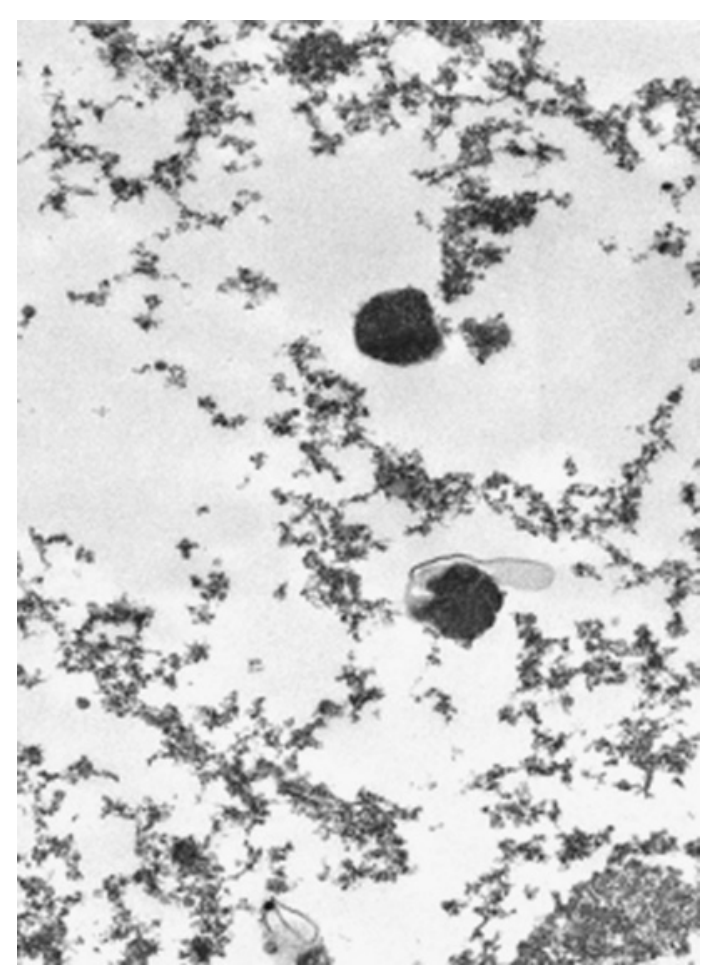

Figure 5 Ultrastructural examination showing some dense cores of $250 \mathrm{~nm}$ diameter in the cytoplasm of the tumour cells.

LCNECs of the lungs ${ }^{2}$ and stomach ${ }^{6}$ usually indicate a poor prognosis due to higher grade malignancy than conventional lung cancers and gastric adenocarcinomas. Certainly, LCNECs usually show a high proliferation rate and numerous mitotic figures. All salivary LCNECs also have poor outcomes. ${ }^{10-13}$ In this study, however, three of the eight cases of M-LCNEC (cases 2, 3 and 4) were fatal. Most of the remaining patients have been disease-free during a follow-up period of 24-108 months. As case 3 presented with particularly marked NE features (strong and diffuse immunopositivity for all three NE markers and for TTF-1), increased NE features are likely to be related to a poor outcome. Seven of the eight cases of M-LCNEC (87.5\%) had many regional lymph node metastases, suggesting that M-LCNEC is likely to show a higher rate of lymph node metastasis than conventional squamous cell carcinomas of the head and neck. All the cases of M-LCNEC in the present series were men and aged $>65$ years. Moreover, the supraglottis and the tongue base were the common primary sites. The supraglottis is the most common site of carcinoids in the head and neck region ${ }^{18-20}$ and therefore such an anatomical location may be important for tumorigenesis of M-LCNECs. Although M-LCNEC displays such clinical features, further follow-up studies are needed to confirm the adverse prognosis of M-LCNEC. A recent report indicated a poor prognosis of LCNEC of the larynx,$^{15}$ and Japanese patients with M-LCNEC also had a relatively poor prognosis.

In one case of M-LCNEC occurring at the tongue base which was previously reported (case 2), ${ }^{14}$ the normal basal cells adjacent to the tumour were immunopositive for CD56 and $\mathrm{N}$-cadherin but not for ChA and Syn. Some basal cells in the mucosa of the head and neck regions have NE or neuroectodermal differentiation, although their morphology could not be distinguished from that of other basal cells of the squamous epithelium. Such a finding indicates that some basal cells expressing CD56 and $\mathrm{N}$-cadherin are related to the origin of 


\section{Take-home messages}

- Large cell neuroendocrine carcinoma (LCNEC) rarely occurs as a mucosal lesion in the head and neck regions (M-LCNEC).

- M-LCNEC was shown to be immunopositive for two or three neuroendocrine markers (CD56, chromogranin-A and/or synaptophysin).

- LCNECs of the lungs and other organs are usually high-grade malignancy, and M-LCNEC of the head and neck has a relatively poor prognosis.

M-LCNEC and are scattered in the mucosa of the head and neck regions.

In summary, this study provides basic information on the clinicopathological characteristics of M-LCNECs of the head and neck regions. The results provide compelling evidence that M-LCNEC is a distinct clinicohistopathological entity. Immunohistochemistry for NE markers and p63 appears to be very useful for the diagnosis of M-LCNEC.

Acknowledgements The authors thank Mr Isamu Hayashi, Mr Yoichi Watanabe, Ms Sachiyo Oono, Ms Kaori Nagata, Mr Hiroshi Tashiro, Mr Koji Muramatsu, Mr Masatake Honda, Ms Chiho Tashiro, Mr Takuya Kawasaki and Ms Eri Yamazaki, the staff of the Pathology Division, Shizuoka Cancer Center Hospital, Shizuoka, Japan, for excellent technical assistance. We are also grateful to Ms Minako Ishii, secretariat of the Pathology Division, Shizuoka Cancer Center Hospital, Shizuoka, Japan, for her valuable help in the preparation of this manuscript.

Contributors All authors contributed equally to this article.

Competing interests None.

Patient consent Obtained.

Ethics approval Approval of the study was granted by the Institutional Review Board ad Shizuoka Cancer Center Hospital and Research Institute.

Provenance and peer review Not commissioned; externally peer reviewed.

\section{REFERENCES}

1. Brambilla E, Pugatch B, Geisinger K, et al. Large cell carcinoma. In: Travis WD, Brambilla E, Mulller-Hermelink HK, et al, eds. World Health Organization Classification of Tumours: Pathology and Genetics of Tumours of the Lung, Pleura, Thymus and Heart. Lyon: IARC Press, 2004:45-50.

2. Jiang SX, Kameya $T$, Shoji M, et al. Large cell neuroendocrine carcinoma of the lung: a histologic and immunohistochemical study of 22 cases. Am J Surg Pathol 1998:22:526-37.

3. Chen KT. Composite large-cell neuroendocrine carcinoma and surface epithelialstromal neoplasm of the ovary. Int J Surg Pathol 2000;8:169-74.

4. Eichhorn JH, Lawrence WD, Yong RH, et al. Ovarian neuroendocrine carcinomas of non-small-cell type associated with surface epithelial adenocarcinoma. A study of five cases and review of the literature. Int J Gynecol Pathol 1996;15:303-14.
5. Jones K, Diaz JA Donner LR. Neuroendocrine carcinoma arising in an ovarian mucinous cystadenoma. Int J Gynecol Pathol 1996;15:167-70.

6. Jiang SX, Mikami T, Umezawa A, et al. Gastric large cell neuroendocrine carcinomas: a distinct clinicopathologic entity. Am J Surg Pathol 2006;30:945-53.

7. Matsui K, Jin XM, Kitagawa M, et al. Clinicopathologic features of neuroendocrine carcinomas of the stomach: appraisal of small cell and large cell variant. Arch Pathol Lab Med 1998;122:1010-17.

8. Evans AJ, Al-Maghrabi J, Tsihlias J, et al. Primary large cell neuroendocrine carcinoma of the urinary bladder. Arch Pathol Lab Med 2002:126:1229-32.

9. Hailmariam S, Gaspert A, Komminoth $P$, et al. Primary, pure, large-cell neuroendocrine carcinoma of the urinary bladder. Mod Pathol 1998;11:1016-20.

10. Hui KK, Luna MA, Batsakis JG, et al. Undifferentiated carcinomas of the major salivary glands. Oral Surg Oral Med Oral Pathol 1990;69:76-83.

11. Nagao T, Sugano I, Ishida $Y$, et al. Primary large-cell neuroendocrine carcinoma of the parotid gland: immunohistochemical and molecular analysis of two cases. Mod Pathol 2000;13:554-61

12. Casas $\mathbf{P}$, Bernaldez $\mathrm{R}$, Patron $\mathrm{M}$, et al. Large cell neuroendocrine carcinoma of the parotid gland: case report and literature review. Auris Nasus Larynx 2005;32:89-93.

13. Larsson LG, Donner LR. Large cell neuroendocrine carcinoma of the parotid gland: fine needle aspiration, and light microscopic and ultrastructural study. Acta Cytol 1999:43:534-6.

14. Kusafuka K, Asano R, Kamijo T, et al. Large cell neuroendocrine carcinoma of the tongue base: case report of an unusual location with immunohistochemical analysis Int J Oral Maxillofac Surg 2009;38:296-9.

15. Lewis JS Jr, Spence DC, Chiosea S, et al. Large cell neuroendocrine carcinoma of the larynx: definition of an entity. Head Neck Pathol 2010;4:198-207.

16. Travis WD, Linnolis RI, Tsokos MG, et al. Neuroendocrine tumors of the lung with proposed criteria for large-cell neuroendocrine carcinoma: an ultrastructural, immunohistochemical, and flow cytometric study of 35 cases. Am J Surg Pathol 1991;15:529-53

17. Greene L, Brundage W, Cooper K. Large cell neuroendocrine carcinoma of the larynx: a case report and a review of the classification of this neoplasm. J Clin Pathol 2005:58:658-61.

18. Hartley C, Brizgalis AR, Lyons TJ, et al. Neuroendocrine carcinoma of the larynx. J $R$ Coll Surg Edinb 1996;41:333-5.

19. Wenig B, Gnepp D. The spectrum of neuroendocrine carcinomas of the larynx. Semin Diagn Pathol 1989:6:329-50.

20. Barnes L. Neuroendocrine tumours. In: Barnes L, Eveson JW, Reichart P, et al, eds World Heath Organization Classification of Tumours: Pathology and Genetics: Head and Neck Tumours. Lyon: IARC Press, 2005:135-9.

21. Kusafuka K, Ferlito A, Lewis JS Jr, et al. Large cell neuroendcrine carcinoma of the head and neck. Oral Oncol 2012;48:211-15.

22. Ferlito A, Silver CE, Bradford CR, et al. Neuroendocrine neoplasms of the larynx: an overview. Head Neck 2009;12:1634-46.

23. Socia E, Kloppel G, Sobin LH. World Health Organization International Histological Classification of Tumors. Histological Typing of Endocrine Tumors. 2nd edn. New York: Springer, 2000.

24. Ferlito KO, Ferlito A, Rinaldo A. Neuroendocrine carcinomas of the larynx: what do the different histologic types really mean? Eur Arch Otorhinolaryngol 2010:267:1323-5

25. Kusafuka K, Hirobe $\mathrm{K}$, Wato $\mathrm{M}$, et al. CD56 expression is associated with neuroectodermal differentiation in ameloblastomas: an immunohistochemical evaluation in comparison with odontogenic cystic lesions. Med Mol Morpho 2011:44:79-85.

26. Strum N, Rossi G, Lantuejoul S, et al. Expression of thyroid transcription factor-1 in the spectrum of neuroendocrine cell lung proliferations with special interest in carcinoids. Hum Pathol 2002;33:175-82.

27. Strum N, Lantuejoul S, Laverriere $\mathbf{M H}$, et al. Thyroid transcription factor 1 and cytokeratin 1,5,10,14 (34 $\beta E 12)$ expression in basaloid and large-cell neuroendocrine carcinomas of the lung. Hum Pathol 2001;32:928-5. 